This document is the accepted manuscript version of the following article:

Shevchik, S. A., Meylan, B., Violakis, G., \& Wasmer, K. (2019). 3D reconstruction of cracks propagation in mechanical workpieces analyzing non-stationary acoustic mixtures. Mechanical systems and Signal Processing, 119, 55-64.

https://doi .org/10.1016/j.ymssp.2018.09.022

This manuscript version is made available under the CC-BY-NC-ND 4.0

\title{
license http://creativecommons,org/licenses/by-nc-nd/4.0/ \\ 3D reconstruction of cracks propagation in mechanical workpieces analyzing non-stationary acoustic mixtures
}

\author{
Sergey A. Shevchik ${ }^{1}$, Bastian Meylan ${ }^{1}$, Georgios Violakis $^{1}$ and Kilian Wasmer $^{1 *}$ \\ ${ }^{1}$ Federal Laboratory for Materials Science and Technology, Thun, Switzerland
}

\section{Abstract}

Cracks are one of the main causes of solid bodies weakening and are a hidden threat for mechanical systems. Acoustic emission is an indicator of cracks initiation and propagation and is widely exploited for the localization of cracks in non- or weakly-transparent environments. The present study enriches the functionality of multiple existing algorithms in crack localization using acoustics. The novelty is in the reconstruction of the complex geometry of the crack path and tracking its propagation in time, whereas existing methods focus only on the localization of the crack without any information about its geometry. The algorithm uses sparse acoustic signal representations as relative energies of the narrow frequency bands, extracted with the $M$-band wavelet transform. Non-linear independent component analysis is applied to de-mix the recorded acoustic signals into a number of separate acoustic patterns. Furthermore, a triangulation, using the time delay arrivals, is applied for each of such patterns separately, thus extracting multiple individual emittance sources. The algorithm was tested using synthetic data that replayed various scenarios of crack propagation together with different detector arrays configurations and its behavior was analyzed. Additional verification with real-time data was carried out by analyzing signals from crack propagation in glass along a known programmed path. The acoustic data was recorded with four fiber Bragg gratings. In both cases, the algorithmic framework showed a high efficiency in recovering the geometrical configuration of the crack.

Index Terms - Acoustic emission, source localization, cracks, non-stationary mixtures, independent component analysis.

\section{Introduction}

The diagnosis and prevention of the catastrophic failure of workpieces in mechanical systems is a critical task in fracture mechanics and is of utmost importance in numerous applications [1]. The nature of cracks is caused by a release of locally accumulated mechanical stresses that exceed the fracture toughness $(K c)$ of the material. The exact distribution of those stresses inside a material is impossible to forecast as their re-distribution in time has a stochastic

${ }^{*}$ Corresponding author, Feuerwerkerstrasse 39 CH-3602, Switzerland, email: kilian.wasmer@empa.ch 
nature $[1,2,3]$. The occurrence of cracks results in the creation of new free surfaces inside a solid medium, which after a certain time reach a critical size and cause a sudden fracture of the material [1,2]. This leads to extensive cost in terms of either damages, delay in production lines or even injury of people [1,2]. For this reason, monitoring of cracks has been in the focus of researchers since the first work of Griffith in this field since 1920 [3]. Despite all the efforts, predicting the location of the crack initiation, direction and velocity of its propagation remains unsolved until today. Consequently, precise diagnostic systems that monitor this process and evaluate the possible damage are required.

Acoustic emission (AE) is a relevant method for cracks monitoring mainly due to its applicability to non-transparent media and is, nowadays, a standard diagnostic tool in many applications [4-6]. AE derives from elastic waves deformations, generated by the release of the locally stored stresses mentioned above [4]. This nature of AE allows to correlate part of its energy with the induced damages [4,7]. Another advantage of AE is in the possibility to spatially localize the cracks in non-transparent solid environments [7-10]. This information is crucial for many mechanical systems, where the geometrical configuration of the crack propagation path is a key factor in estimating the workpiece lifetime [11].

At present, the existing crack localization methods are mainly based on time delay of arrival (TDA) of the acoustic wave [7-11]. In general, this method exploits the known propagation speed of AE inside the medium and known relative positions of the detectors that are used for further triangulation. Reconstruction of the geometrical configuration of the crack path using TDA is also possible by triangulating the individual patterns, extracted from received AE signals. The usage of the individual patterns also allows to replay the propagation of the crack in the time domain $[9,12,13]$. However, despite the apparent simplicity, this approach remains a complicated undertaking.

The search of pattern correspondences within several acoustic signals is a non-trivial task. This is mainly due to the distortions in the received signals due to environmental factors, presence of noises and complex mixture of individual AE patterns between each other $[12,14]$. Several methods for pattern correspondences search exist and they are based on cross-correlation and statistical analysis $[7,13,15,16]$. The robustness of those methods can be increased by noise suppression in the detected signals $[15,16,17]$, while the environmental distortions can be compensated by solving inverse problems of the acoustic wave propagation inside a medium $[12,14,18]$. Both approaches may reduce the number of outliers in the pattern correspondences but require a detailed knowledge about the environmental properties and/or noise characteristics, which are not always available. In real life applications, this brings inaccuracies in those algorithms performance in unknown environments. Another constraint of the existing methods is in the reconstruction of the crack propagation path that usually takes place in several directions with individual propagation velocities. In this case, the received signals are complex mixtures of multiple AE patterns with unknown content. Those patterns are produced by an unknown number of cracks sub-paths that have different 
localization. A mixing of the patterns takes place with random drifts relatively to each other, due to the presence of noise and environmental distortions. Today, no algorithm exists that allow reconstructing the crack path under such circumstances. At the same time, knowing the geometry of crack path is crucial to predict the lifetime of mechanical components [19].The present study is a supplement to existing studies on crack localization that aims to remove the constraints of existing methods, namely: i) operate without prior knowledge on the environment and ii) reconstruct the crack propagation path geometry in case of several propagation directions and track their changes in time. This is achieved by de-mixing the received AE signals into individual patterns using non-linear independent component analysis (ICA).

ICA [20] is a statistical method that aims to decompose a given signal into several subcomponents with a maximum independence, relying on the non-Gaussianity in their distributions [20]. As mentioned above, in this study, the received AE signals are considered as mixtures of multiple separate acoustic patterns of non-stationary nature and the objective of ICA applications is to define the mixing law.

The attractiveness of ICA towards the crack initiation and/or propagation monitoring problems is in the possibility to operate on signals without prior knowledge about their characteristics. The released AE from cracks includes information about the local structural degradation that is locally unique [21] and this is a direct task for ICA. In addition, ICA allows compensating the non-linear environmental distortions and noises within a single framework. The known limits of this method are in operating with non-Gaussian probability distributions and impossibility to estimate the order and the variance of the sub-components [20-21]. This causes redundancy in computations so that the usage of ICA is an open topic regarding crack problems. Our motivation for employing ICA in this work was given by the research of Jiao et al. [5]. The authors showed that the AE induced by a crack is a mix of several modes that differs from each other in a statistical way and are separable. In Mostafapour et al. [18], it was demonstrated that the signal content and the environmental distortions in the AE possess several unique characteristics that can be differentiated. Therefore, the present work investigates the feasibility of spatial localization of crack initiation and/or propagation employing mathematical tools [20], keeping the physical explanation of the AE content outside the scope of this work.

The article is divided into four sections. Section Crack initiation/propagation induced AE signals and test datasets has three sub-sections. The first one describes the particularities of crack induced acoustic signals. The second explains the creation of synthetic dataset (artificial dataset) to check our algorithm. The last describes the acquisition of the real life dataset. The section Signal processing incorporates the description of sparse signal representations using $M$-Band wavelets and the ICA method. The section Results and discussion presents the tests on both synthetic and real life data and the evaluations of the algorithm. The conclusion summarizes the main results and findings as well as perspective of future work. 


\section{Crack initiation/propagation induced AE signals and test datasets}

\section{Crack initiation and/or propagation induced AE signals}

Acoustic emission from crack initiation and/or propagation has been investigated in the last several decades and is actively used in a number of field applications [19]. A typical example is shown in Fig.1, where Fig.1,a is an acoustic emission obtained from the crack in Fig.1,b, in which its path is presented. The crack AE signals are typically long tailed and are characterized by a rapid increase in the AE intensity during the crack initiation, followed by a gradual attenuation during its propagation $[11,15,16,19]$. It is interesting to note that the shape of the signal in Fig. 1, a remains similar for most of the materials $[11,15,19]$. This aspect is efficiently exploited in practice where this distribution is often approximated to be an inverse Gaussian [19]. Several parameters of such signals, such as the wave rise time, attenuation velocity, peak position and amplitude, etc. are used for damage estimation [19]. Nevertheless, the AE content in Fig.1,a is of higher complexity as can be seen in the multiple fluctuations of the signal. The analysis of this content in terms of dynamics was under active research and an overview can be found in [22]. This study, exploits the results reported by Ibrahim [22], where the dynamics of the crack growth is considered as Itô process. In this work, we extend those findings by employing ICA for non-linear de-mixing of AE patterns inside signals analogues to the one from Fig.1,a.
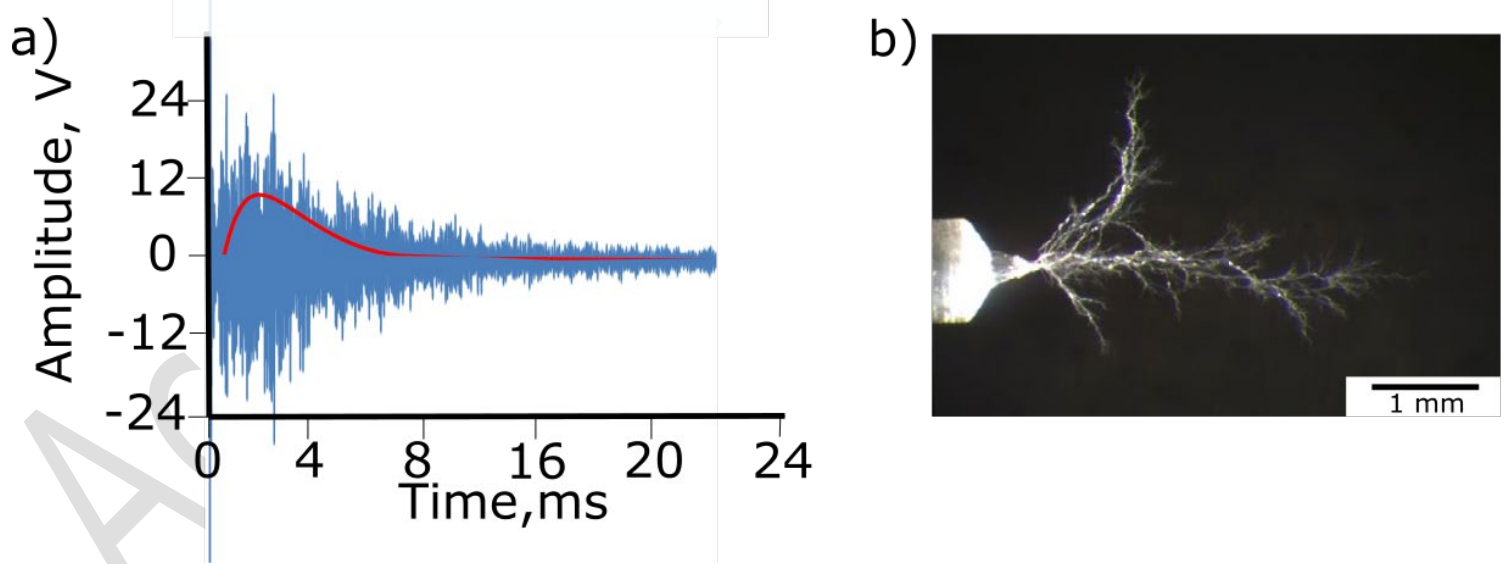

Fig. 1. (a) Typical acoustic emission signal emitted during a crack initiation and propagating in acrylic glass, recorded using a $1 \mathrm{MHz}$ sampling rate. The red line shows an approximation of the intensity using an inverse Gaussian distribution; (b) the image of the crack path, where the propagation took place in several directions, resulting a branchy structure of the crack path. 


\section{Synthetic dataset}

The evaluation of the algorithm was made using both, synthetic and real life datasets. First, the synthetic dataset (artificial dataset) was created to simulate the dynamics of crack propagation. One representative scenario of crack propagation can be seen in Fig.2, a. In this setup, the crack was initiated at a specific point, marked as root in Fig.2,a, and further propagated along a branching path. This path was constructed by splitting each previous path segment into two new ones at a given time and the location of the split hereafter is referred to as splitting node. At the same time, the new paths were characterized by new random propagation directions and length. The final amount of path segments in the tree was determined as $2 r$, where $r$ is the number of splitting nodes for each individual tree branch, starting from the root and is hereafter referred to as the split nodes number. The example in Fig.2,a includes 5 levels of splitting nodes in each branch. The propagation velocity of crack was kept constant and the number of the splitting nodes varied thus affecting the final number of path branches. For the synthetic dataset, the number of detectors, their relative positions, as well as the number of the splitting nodes was changed according to the user setup. Each new crack propagation scenario was characterized by random positions of the split nodes relatively to the detectors, the propagation directions and the number of the branches (e.g. split nodes number).

Each individual segment of the path tree, constrained by a pair of neighboring splitting nodes, was put in correspondence with a unique acoustic pattern. Each such pattern was reconstructed with an inverse Fourier transform of a unique frequency band, generated randomly. The signal, received by each detector, was a mixture of such multiple patterns, constructed according to the following term: $y_{i}(x)=\sum_{j} a_{j}(x) d t_{i j}+G$, where $y_{i}$ are the received signals at the detector $i, a_{j}$ are the patterns generated according to the technique described above, $j$ indicates the branch of the crack path like the ones in Fig.2,a, $d t_{i j}$ are the specific time delays of the individual patterns that are derived from the relative location of the pattern source and the given detector, $G$ is an added mean zero Gaussian noise. As seen from mixing rule, the TDA includes the specific arrival time of each individual pattern with respect to the position of each sensor relatively to the position of the individual segment of the path tree. The velocity of acoustic wave propagation inside the medium was assumed to be constant and was not affected by the local non-uniformities of the medium. The application of the developed algorithm to synthetic dataset aimed to recover all approximates of the individual AE patterns from the resultant mixture and localizes their positions.

In Fig.2,b, an example of the visual representation of the resultant signal is presented. This signal is a mixture of individual unique AE patterns, produced by each path segment from the scenario presented in Fig. 2,a. The Fourier bands of each individual pattern included inside the signal from Fig.2,b included two thousand frequencies. The characteristics of the patterns were generated uniquely for each segment of the crack path from Fig.2,a. As can be seen from 
the resultant signals in Fig.2,b, their characteristics are close to the real life ones shown in Fig.1,a. Both signals have a rapid release of energy at the beginning with attenuation while propagating further.

a)

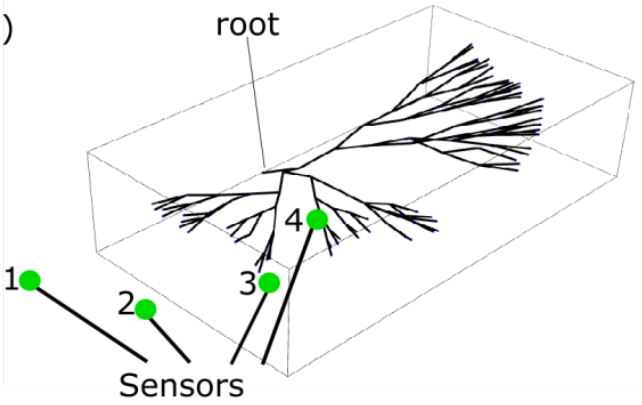

b)

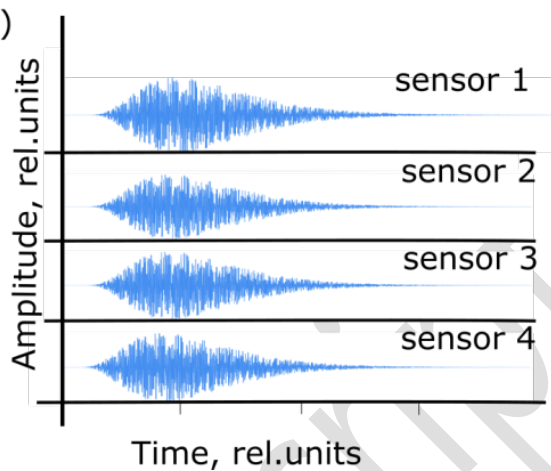

Fig.2 (a) The spatial configuration of the crack path inside the medium, where the propagation starts from the root and the entire tree includes four levels of splitting nodes. The boundaries mark the volume, in which the crack was created; (b) the resultant acoustic emission that was received at one of the sensors and is a mixture of many unique patters of the crack path from (a). The sensor number in (a) corresponds to the ones in (b). The sensors, in this case, are placed over the crack, in the plane of the upper border of the crack volume, bordered by the cube.

\section{Real life dataset}

The real life signals were obtained by recording airborne AE during the initiation and propagation of a crack inside a glass plate. The glass plates had dimensions of $75 \mathrm{~mm}$ by 25 $\mathrm{mm}$ and $1.5 \mathrm{~mm}$ thickness. Each glass plate had an amorphous structure, meaning it did not possess any preferential crystallographic direction for crack propagation. A scratch was made in the middle with a diamond pen, as indicated by a white arrow in Fig.3,a. The scratch induced an initial crack in order to force the crack to propagate along this path when breaking the glass plate into two pieces and so to reduce the localization problem to $2 \mathrm{D}$ case as shown in Fig. 3,b. The glass plates were placed with the scratch facing downwards in a special holder surrounded by four acoustic sensors. Those sensors were not in contact to the glass plate but they were placed at a certain distance from the glass plate center as well as radially symmetric to it as can be observed on the image of the entire setup in Fig. 3,c. In order to initiate and propagate the initial crack, a metal pin was pressing slowly the glass plate based on a threepoint bend test. The breakage of the glass plate took place in two stages. First, a fast crack initiation took place. Then, the crack propagates at high speed through the glass plate to get two pieces. This is a well-known cracking behavior in brittle materials [23-25]. The records of the signals were triggered when the released airborne AE energy exceeded the predefined threshold at one of the four record channels. The exact AE threshold was defined from a preliminary study of crack initiation and propagation on similar glass plates. 
Fiber Bragg gratings (FBG) were used in this contribution for sensing the airborne AE due to their extreme sensitivity. FBGs are interferometric structures, imprinted inside an optical fiber core [26]. The variations of the refractive index inside this structures has a fix period $\Lambda$ and provides a reflection of selected wave lengths dependent on the period $\Lambda$ according to the relation [26]:

$$
\lambda_{b}=2 \mathrm{n}_{e f f} \Lambda
$$

where $\lambda_{b}$ is the reflected wavelength (called Bragg wave length), $n_{\text {eff }}$ is the refractive index of the fiber core (i.e. the refractive index of the core for a particular mode, typically the LP01). The arrival of acoustic or pressure waves on the sensor induces a local elongation of the fiber which affects the periodicity $\Lambda$ and $\lambda_{b}$ according to Eq. (1). This causes modulations in the reflected light, a wavelength of which is chosen in the neighborhood of $\lambda_{b}$. More details on this technology can be found in the review of Kulchin et al. [26]. The FBGs involved in this work possessed a reflectivity of $65 \%$ at $\lambda_{b}=1546.5 \mathrm{~nm}$. The modulations of the reflected light were detected by photodiodes with the sensitivity in the spectral range of $1.1-2.5 \mu \mathrm{m}$, connected to a 4 channel oscilloscope Lecroy HDO 4054 (Lecroy, USA) with a sampling rate per channel of $1 \mathrm{MHz}$. The orientation of the FBGs was radial towards the center of the glass plate providing the maximum response to airborne AE. The sensors were hanged in the air with the same pre-strain on all sensors and without any contact to the surrounding surfaces. This was done using plastic holders that were placed with the defined known relative positions and can be observed in Fig.3, c. The received signals were with a $50 \mathrm{~dB}$ hardware amplification on the photodiode side. The detection of the signals was carried out using a trigger that was set to exceed the FBG signal range of $0.1 \mathrm{~V}$. The received FBG signal varied in the range of $0-3 \mathrm{~V}$. The signals were recorded without any hardware filtering.

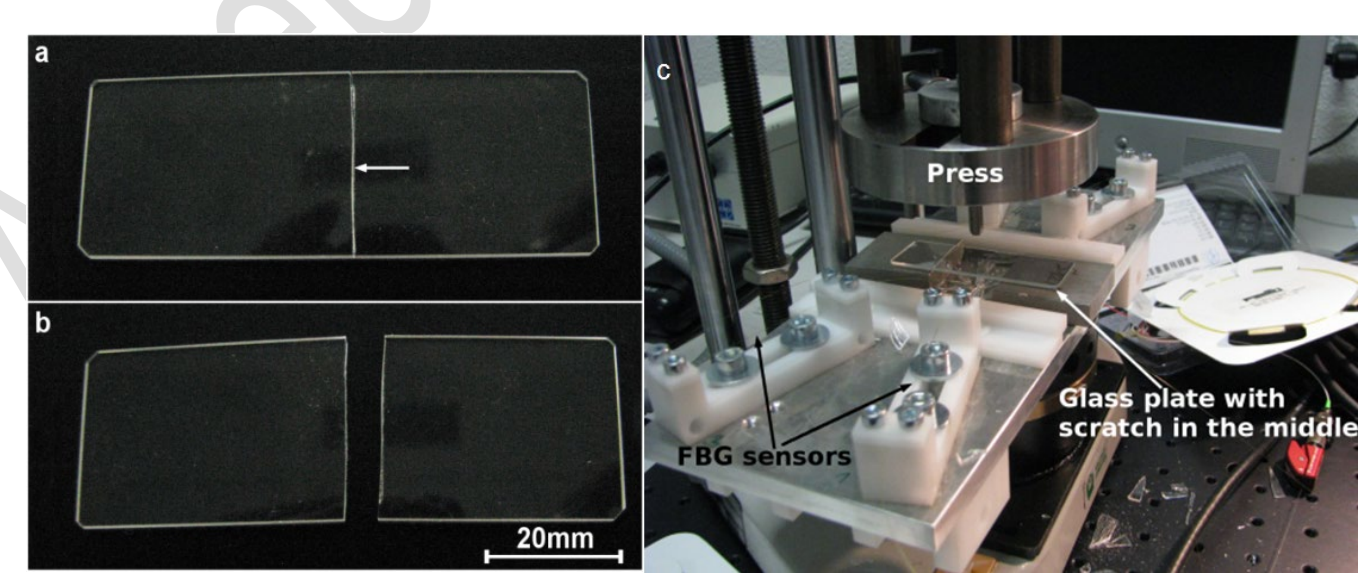

Fig. 3 Thin glass plate used for the experiment (a) before breakage and (b) after. The arrow in (a) shows the prescratching of the plate to guide the crack propagation; (c) setup used to break the glass plates and record the acoustic emissions with four FBG sensors placed at known positions around the sample. 


\section{Signal processing}

\section{Sparse signals representation using $M$-band wavelet transform}

In this work, the sparse signals representations with the relative energies of the narrow frequency bands were used. The latter were obtained from $M$-band wavelet transform (MWT). MWT is an extension of traditional wavelet techniques [27] that gives several advantages. It provides a more detailed tiling of the time-frequency space and employs several wavelets operating at different subspaces of the input signals, thus, resulting in the reduction of artifacts in data decomposition [28]. For these reasons, $M$-band wavelets were chosen to process the real life data in this work.

The principles of MWT can be interpreted as passing the signal through multichannel filters [28]:

$$
\begin{array}{cc}
\varphi_{j}(n)=\sum_{n} h_{0}(k) \sqrt{2} \varphi(2 n-k), \quad k \subset Z \\
\psi_{j i}(n)=\sum_{n} h_{M-1}(k) \sqrt{2} \psi(2 n-k), \quad k \subset Z
\end{array}
$$

where $\varphi(n)$ is a scaling function, $j$ is a scale level, $\psi()$ is a wavelet function, $h_{0}, h_{M-1}, h_{M-2}$ are the low, narrow and high pass filters, respectively, $n$ are the samples and $k$ is a shift. The application of Eqs (2) and (3) results in the extraction of the low, narrow and high frequency bands that are localized in the time-frequency domain and are described by the coefficients $\left\{d_{m}\right\}$. The analysis at several scales is carried out using a sequential filtering of the low frequency content from the previous transform according to the scheme presented in Fig.4,a. This results in the tiling of the time-frequency domain into a pyramidal structure of narrow frequency bands that may be used to characterize the energy re-distribution in time. The energy of the individual frequency band is a suitable characteristic for the computations and is defined as [28]:

$$
E_{j, m}=\int\left|d_{j, m}(t)\right|^{2} d t=\sum_{k}\left|d_{j, m}\right|^{2}
$$

where $d$ are the wavelet or scale function coefficients extracted from Eqs (1) and (2). Following Eq. (3), the relative energies are the normalized version of the sub-band energies which are defined as:

$$
\rho_{\text {norm } j, m}=\frac{E_{j, m}}{E_{j}}
$$

where $E_{j}=\sum_{k=0}^{M^{j}}\left|E_{j, m}\right|^{2}$ is the total energy accumulated in all frequency bands. In this contribution, the collection of the relative energy from Eq. (5) was used for the sparse signal 
representation. A four band filter was employed for the signals decomposition which was taken from Lin et al. [29].

The filtering of the data using the results from the MWT decomposition is provided by the possibility to choose the scale range so that it is possible to zoom into the most informative frequency spectral range. The frequency bands from all scales, bounded by a fixed time span as shown with the red frame in the sonogram in Fig.4,b, are the unique characteristic of the individual AE patterns. Those represent the distribution of energy between the different bands in the time-frequency domain. In this study, the received signals from the detectors were decomposed using the MWT and the pattern correspondences were searched using the wavelet sonograms. Each of such patterns is a mix of several sub-patterns arriving from the different AE sources, generated by different branches of the crack path.

a)

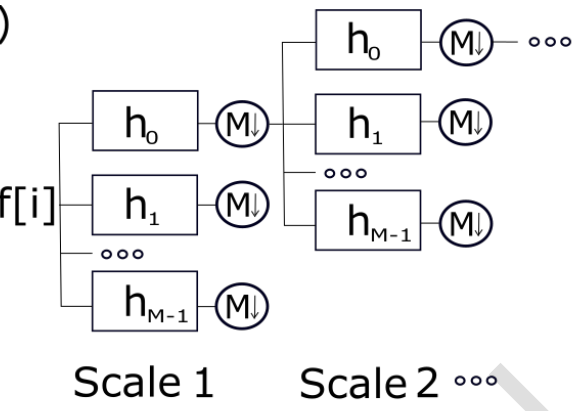

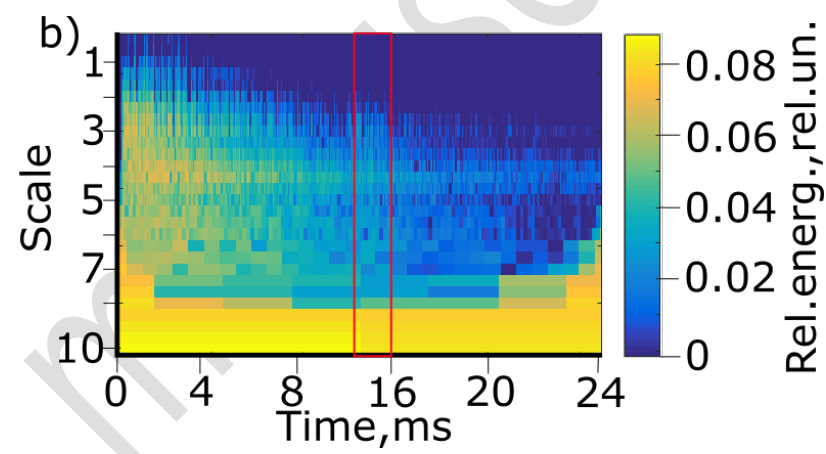

Fig. 4. a) $M$-band wavelet transform, where $h_{0}$ and $h_{1}-h_{M-2}$ and $h_{M-1}$ are the low, narrow and high pass filters respectively; b) $M$-band sonogram for the signal from Fig.1,a, in which the red frame in Fig.4,b of a fixed time span denotes a separate pattern.

\section{Independent component analysis (ICA) for individual patterns recovery}

Blind source separation using ICA is a wide research area [20] that aims to recover the hidden sub-components of a given signal using statistical independency principles. Those principles are based on the central limit theorem [30], which claims that a number of joint probability distributions from several statistically independent variables are closer to a Gaussian distribution than the individual distributions of those variables. Consequently, the measure of the independency of the sub-components is their non-Gaussianity [30]. The general problem formulation is in collecting a number of observations $y=\left\{y_{i}\right\}$, where $i=1, \ldots, N$ and corresponds to the number of detectors. Each $y_{i}$ consists of a mixture of unobservable subcomponents $z=\left\{z_{j}\right\}$, where $j=1, \ldots, M$, generated by $M$ unrelated processes. The mixing rule is expressed as $y=A z$ [20], where $A$ is a $N \mathrm{x} M$ matrix containing the information about the mixing proportions. The statistical independency of $z_{j}$ leads to: $P(z)=\Pi p\left(z_{j}\right)$, where $P(z)$ is a joint probability density and $p\left(z_{j}\right)$ is the density probability for the individual sub-component $j$. In our setup, each observation corresponds to an AE signal, received at each of the $N$ 
detectors, and $z$ are the individual unique acoustic patterns with a different spatial localization along the crack path. The de-mixing of the AE mixtures is carried out by computing the matrix $W=A^{-1}$ such that:

$$
z^{* T}=W y^{T}
$$

and $z_{j}^{*}$ is an approximation of $z_{i}$. The solution for $W$ is obtained via an iterative optimization, in which the measure of the non-Gaussianity in ICA are the fourth order moment (i.e. kurtosis), mutual information or other parameters [31].

We have mentioned that the problem of applying the general ICA to the crack problem is the non-stationary nature of the AE. This indicates that the number of sub-components is not constant and the AE patterns have unknown time delay arrival (TDA) drifts for different individual AE patterns. Both factors depend on the geometrical configuration of the crack path. To overcome this difficulty, a non-linear version of an ICA was employed that recovers a collection of the momentary mixing rules $A$ that may further be gathered to recover the evolution of $z$ during the entire process. As already said, we exploited the possibility to approximate the crack initiation and/or propagation developed by the Itô setup [22] which was combined with a specialized ICA from Singer and Coifman [32]. Its additional advantages are in using diffusion maps that have low sensitivity to noises and the possibility to recover the non-linear transformations of $z_{j}$ due to the environmental distortions for real life crack monitoring applications.

In the Itô setup, the observations $y$ are the non-linear transformations of $z_{j}$ expressed as: $y=f\left(z_{1}, \ldots, z_{M}\right)$. The process develops in time and the momentary values $z_{j i}$ are formed as:

$d z_{j i}=a_{j i}\left(x_{j i}\right) d t+b_{j i}\left(x_{j i}\right) d n_{j i}$, where $a_{i j}$ are the drifts, $b_{j i}$ are the noise coefficients and $n_{j i}$ are the uncorrelated white noises [32]. The search for the independent sub-components is carried out with the unknown transformation $f()$, drifts $a$, noise levels $b$. The adaption of the solution of Singer and Coifman [32] for the sparse representation using the narrow frequency bands is performed as follows.

The received AE signals $y$ and each $y_{i}$ is divided into a sequence of separate AE patterns $p_{j i}=d y_{j i}=f\left(d z_{1 i}, \ldots, d z_{M i}\right)$, where $j=1, . ., M$ is the localization of the pattern in the time domain, $i=1, \ldots, N$. The visual representation of such patterns with a fixed time span $i$ is schematically shown in Fig. 4,b. Following Singer and Coifman [32], the covariance Cov $_{j}$ for the patterns $p_{j i}$ at fixed $j$ is computed according to: $\operatorname{Cov}_{j}=J J T$ with the size of $\operatorname{Cov}_{j}$ as $N x N$ and $J$ as the Jacobi matrix. Then, the normalized Laplacian is computed as: $L_{j}=D^{-1 / 2} W D^{-1 / 2}$, where $W$ is the affinity matrix obtained from $J$ using the following term [32]:

$$
W_{m k}=\exp \left[-\frac{\left\|J^{-1}\left(y_{k}\right)\left(y_{k}-y_{m}\right)\right\|+\left\|J^{-1}\left(y_{m}\right)\left(y_{k}-y_{m}\right)\right\|}{4 \mathrm{e}}\right]
$$

where $k$ and $m=1, \ldots, M$. The approximations $z_{j}^{*}$ of the sub-components $z_{j}$ are recovered by the first $s$ eigenvectors of the constructed Laplacian $L$. All the details of the method used can be 
found in Singer and Coifman [32]. One of the ICA results using the diffusion maps is the recovery of the drifts $a_{j i}$ that are, actually, the delays of the individual patterns $p_{j i}$ so that the TDA triangulation can be applied to localize the pattern sources. It is important to mention that the choice of the time span for the patterns $p_{j i}$ has to be chosen so that it exceeds the TDA of each individual pattern for the different sensors.

It is known that the AE content of an induced crack depends strongly on the configuration of the local grain structure and the residual stresses inside the material medium. Consequently, the natural limits for the ICA method are the statistical independence of the relative energies in the acoustic signals and they are derived from Eq. (6), where the number of the recovered sub-components is linked to the number of observations (e.g. sensors). Some recovery methods for a greater number of sub-components as compared to the number of observations are met in literature [20]. This special case relates to ill - posed problems while the system from Eq. (5) is underdetermined and additional assumptions are needed. Nevertheless, the algorithm performance is analyzed and the results are presented in the section Results and Discussion.

\section{Triangulation using time delay arrival (TDA)}

In our work, the localization problem for each individual pattern correspondence was solved with a standard TDA approach. The position of the single source $j$ is a solution of [33]:

$$
f(x, y, z, t)=\sqrt{\left(x_{j}-x_{s i}\right)^{2}+\left(y_{j}-y_{s i}\right)^{2}+\left(z_{j}-z_{s i}\right)^{2}}-c \cdot\left(t_{j}+\Delta t_{i j}\right)=0
$$

where $\left\{x_{s i}, y_{s i}, z_{s i}\right\}$ are the known positions of the acoustic sensors enumerated with $i=1, \ldots, N, c$ is the sound speed inside the medium, $\left\{\Delta t_{i j}\right\}$ is the TDA from $j$ of the acoustic pattern sensor $I$ and $t_{j}$ is the travelling time. The solution of Eq. (8) is a set of $\left\{x_{j}, y_{j}, z_{j}, t_{j}\right\}$ and we used the Levenberg-Marquardt algorithm [34] for their computations. It was observed that the solution for each individual source $j$ was found, in average, after three iteration steps and the computational errors are discussed in the next section.

All codes, computations and visual representations presented in this work were performed with specially developed tools. All algorithms were programmed in $\mathrm{CH}$ and $\mathrm{C}++$ in Visual Studio 2017. The personal computer, involved for the tests, had i5 processor with $8 \mathrm{~Gb}$ of RAM.

\section{Results and discussion}

\section{Tests on synthetic dataset}

In total, forty five experiments were carried out using synthetic dataset. In each experiment, a new path with an updated geometry, analogous to the one from Fig.2 was generated. All experiments with synthetic dataset were divided into three groups, in which the number of 
branches of the crack path (i) exceeded, (ii) was equal to and (iii) was less than the number of sensors. As explained in the previous section, these combinations lead to overdetermined system in Eq. (6) for case (i) and undetermined system for case (iii). The performance for these cases was specifically examined and compared with case (ii). Additionally, in each group of experiments, the number of sensors varied although their proportions as compared to the number of crack path branches remained the same. The main results for all tests are presented in Table I, where the absolute errors were computed as the Euclidian distance from the ground truth crack path to the 3D position of the individual acoustic source, localized with our algorithm. This distance was computed as a length of a norm from the computed pattern coordinated on the segment of the crack path such as in the example shown in Fig.2,a.

As can be observed from the results in Table I, the increasing complexity of the crack path (characterized by a greater number of the split nodes and path branches as discussed in Section II, B), with a proportional increase of the number of detectors did not affect crucially the accuracy in the crack localization. The corresponding accuracy values for this case are given in rows in Table I. At the same time, the accuracy significantly decreased while increasing the complexity of the tree and, at the same time, decreasing the number of the sensors (the columns in Table I). The undetermined cases, marked as (iii) in Table I, in which the number of sensors was less than the number of branches in the propagation path, shows larger errors than the cases (i) and (ii). For practical applications, this factor limits the accuracy in localizing crack path geometry since the number of the separate branches during the crack propagation is, a priori, unknown and may exceed the number of sensors. Under such circumstances, the higher error rates can be explained by the underdetermined system of equations in Eq. (6), when the number of AE patterns $z_{i}$ is greater than the number of observations $y_{j}$ (for details see Section III,B). This leads to a decrease of the spatial resolution in the crack path computations by averaging the locations from several nearby branches of the propagation path into a single point. This case is illustrated by the example in Fig. 2,a where the branches structure of the tree, that corresponds to the last part of the propagation path, will be averaged into a lower number of branches, which does not exceed the number of detectors.

For case (i) the number of sensors exceeds the number of sub-components that over determines the system in Eq. (5). In this situation, the triangulation defines several acoustic sources with the same coordinates, thus bringing to redundancy in the computations. Tracking of this inside the algorithm may indicate that the number of detectors involved exceeds their critical minimum number for a specific situation. 


\begin{tabular}{l|c|c|c|c}
\hline \multirow{2}{*}{ Case } & \multicolumn{4}{|c}{ Absolute errors \pm standard deviation [mm] } \\
\cline { 2 - 5 } & \multicolumn{4}{|c}{ Number of the splitting nodes in path tree } \\
\cline { 2 - 5 } & 3 & 5 & 7 & 9 \\
\hline (i) $M^{*}<N^{*}$ & $1.4 \pm 0.9$ & $1.5 \pm 1.1$ & $1.0 \pm 0.9$ & $1.2 \pm 1.1$ \\
(ii) $M^{*}=N^{*}$ & $1.3 \pm 0.8$ & $1.4 \pm 0.9$ & $1.0 \pm 0.8$ & $1.4 \pm 0.4$ \\
(iii) $M^{*}>N^{*}$ & $2.3 \pm 1.3$ & $2.0 \pm 1.1$ & $2.4 \pm 1.5$ & $2.3 \pm 1.3$
\end{tabular}

Table I: Accuracy in tracking of crack path

\section{Tracking of cracks initiation and/or propagation with programmable direction in glass}

To get a statistically relevant crack initiation and propagation reconstruction on real data, 10 glass plates were broken and the collected AE data was analyzed. An example for one recorded signal, obtained from an FBG sensor with its corresponding wavelet spectrogram, is presented in Fig. 5,a. As seen from the figure, the typical lifetime of the crack initiation and propagation was observed in the range of $12 \mathrm{~ms}$. The analogous spectrograms were used for each sensor to track the crack propagation path. An example of the reconstruction is presented in Fig.5,b. The process dynamics is encoded in colors according to the color bar on the right side of Fig.5,b, and the entire range fits the crack lifetime of $12 \mathrm{~ms}$. Each sphere encodes a localized separate acoustic source that emits a specific acoustic pattern, extracted using our ICA routine. Taking into account the temperature and the pressure at the time of experiments, the sound velocity for cracks localizations was taken as $343 \mathrm{~m} / \mathrm{s}$. The diameter of each sphere corresponds to the total AE released energy of this specific pattern and the gradient fill indicates the pattern lifetime, according to the time color scale on the right of the figure. The purple line corresponds to the scratch on the surface of the glass that was known from the geometry of the setup. As can be seen from the dynamics presented in Fig. 5,b, the first acoustic pattern was detected at the contact between the pin and the glass plate. Only then, the crack initiated and propagated from this point along the scratch. This also can be tracked from the individual AE sources lifetimes, encoded in colors. The replay of the process dynamics according to the lifetime of the patterns from Fig. 5,b allows to get the information about the spatial localization of the crack. Several acoustic patterns were localized on the side of the plate. However, it was found that those actually corresponded to the contact points of the glass with the underlying basement of the three-point test. Therefore, these patterns represent artifacts and not errors in the crack reconstruction. For this reason, these sources were excluded from the performance evaluation. A full example movie of the crack initiation and propagation dynamics, reconstructed using $\mathrm{AE}$ and our algorithm can be seen at: https://www.empa.ch/web/s204/3Dreconstruction 1.

The same dynamics as in Fig. 5,b was observed for the remaining experiments, where the most of the AE patterns were localized close to or at the scratch location. This was completely in agreement with the real life situation (see the movie), in which none cracks except the 
scratch was observed after glass breakage. A typical example is presented in Fig. 3. The evaluation of the algorithm performance was carried out analyzing the deviation in the distance between the locations of the separate sources relatively to the ground truth position of the scratch. We found that the accuracy based on the set of experiments was $1.9 \pm 1.6 \mathrm{~mm}$. The accuracy was computed as a distance of the norm, from the computed 3D position of the individual AE pattern to the line that represents the scratch on the surface of the glass plate.

The localization error in terms of depth was analyzed separately and was $1.2 \pm 0.6 \mathrm{~mm}$. It is important to note that for the specific case: mean + standard deviation, the majority of the computational reconstruction errors did not exceed the real physical thickness of the glass plate.

It is important to mention that sparse signals representations with wavelet sonograms give the flexibility to adapt the algorithm for various sensors and conditions. This is achieved by tuning a broad spectrum of parameters such as: the number of decomposition levels, the wavelet channels number, decomposition itself (choosing between wavelet packets, dual trees etc.). We also tested the algorithm on synthetic data with the standard two channel wavelet (Daubechies with ten vanishing moments) using wavelet packet transform and observed similar performances.

In essence, the tests of the algorithm using the synthetic signals and the ones obtained from the real life crack propagation events showed a good correspondence with the ground truth. Nevertheless, the approach presented incorporates a built in error source. The narrow frequency band in the signal representation still incorporates the natural ambiguities in the localization in the time domain. It raises errors in estimating the time delays in the patterns correspondences in the collected AE signals. The improvements for algorithm performance are the subject of further investigations.
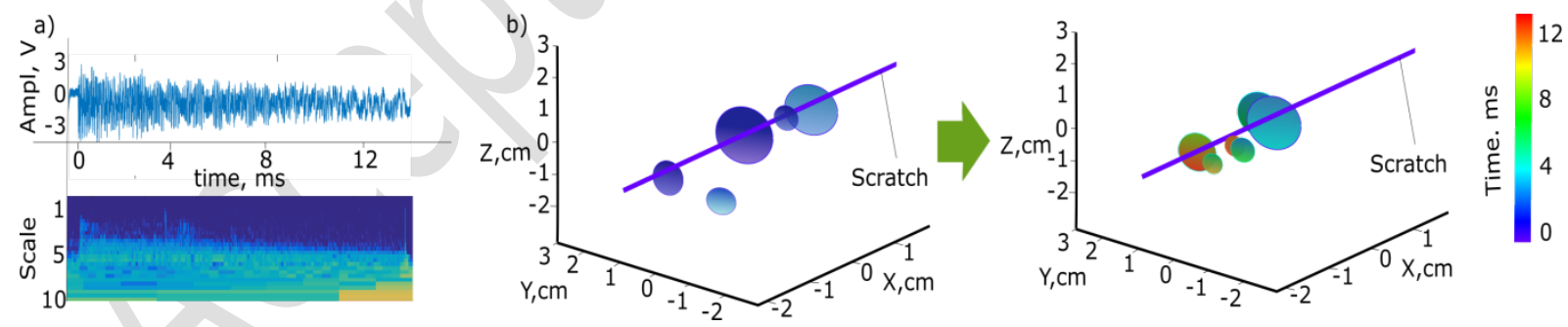

Fig.5 a) (top) Acoustic emission signal recorded from the glass breakage and (bottom) its wavelet spectrogram recorded from one FBG sensor (bottom); (b) the reconstruction of the crack propagation in the time domain, where the time is encoded in colors according to the bar on the right, the gradient fill of the spheres is a pattern lifetime, the diameter of the spheres is the total released acoustic energy and purple line corresponds to the scratch on the glass.

\section{CONCLUSIONS}

This study proposes a new algorithm for tracking cracks initiation and propagation inside a medium. Operating in the time-frequency domain, the technique proposed is able to recover the geometry of cracks even in cases when several propagation paths take place. This brings a 
supplement and enrichment of existing studies on crack path localization and is a novelty in both, the approach and the acquired functionality. The acoustic sensors with known relative positions are registering the AE signals emitted from cracks initiation and propagation inside a non-transparent medium. The received signals were decomposed using $M$-band wavelets with further extraction of the relative energies of the narrow frequency bands. The tracking of the crack initiation and propagation in the time domain is carried out by finding the pattern correspondences within the registered signals and computing the correspondent time delays among those. The search of pattern correspondences is performed by de-mixing the signals using non-linear independent component analysis (ICA). The delays between the specific patterns are computed and a standard time delay arrival (TDA) algorithm is applied to localize the position of the AE source of a specific pattern.

Tests on synthetic and real life data showed that despite the natural limits, ICA is applicable for monitoring crack initiation and propagation problems. Those limits are the statistical independence of the relative energies in the acoustic signals. It is known that the AE content of an induced crack depends strongly on the configuration of the local grain structure and the residual stresses inside the material medium. Consequently, the AE signals induced by a crack initiation and/or propagation fulfil the ICA requirements for the tested materials. In addition, the relative energies of the $M$-band wavelet transform are a suitable framework for signal representation with lower sensitivity to noises as compared to other decomposition techniques.

It was found that an increase in the complexity of the crack path tree (characterized by more complex geometry in crack path), with a proportional increase of the number of sensors did not affect crucially the accuracy in the crack localization. In contrast, the accuracy significantly decreased while increasing the complexity of the crack path geometry while decreasing the number of the detectors. For the real life data, we evaluated the accuracy as $1.9 \pm 1.6 \mathrm{~mm}$.

The results obtained in this study showed that ICA tremendously expands the possibilities of standard TDA approach when analyzing complex distributions of multiple acoustic sources in unknown environments. Such functionality is partially gained due to the natural possibilities of ICA to recover the nonlinearities in signals transforms, caused by the environmental conditions. Additionally, this study shows that crack propagation is characterized by a strong stochasticity in released acoustic signals that can be interpreted in terms of non-stationary mixtures, thus, making ICA applicable. The physical background is in capturing the local degradation features that seem to be unique for each local point in the crack propagation path. This aspect was not clear at the beginning of this study. Although the performance showed the applicability of this method to the crack initiation and propagation problem, its accuracy for heterogeneous materials and in less controllable environment has to be further investigated. 


\section{REFERENCES}

[1] J. Schijve, Fatigue of structures and materials, Springer Science + Business Media, B.V., (2008)

[2] A. Saxena. Nonlinear Fracture Mechanics for Engineers, Boca Raton, Florida: CRC Press (1998).

[3] A. A. Griffith, The phenomena of rupture and flow in solids. Phil. Trans. Roy. Soc. Lond. A221 163 (1920).

[4] M. Ohtsu, K. Ono, The generalized theory and source representations of acoustic emission. Journal of Acoustic Emission 5: 124-33 (1986)

[5] J. Jiao, C. He, B. Wu, R. Fei, X. Wang, Application of wavelet transform on modal acoustic emission source location in thin plates with one sensor, International Journal of Pressure Vessels and Piping 81(5): 427-431(2004)

[6] J. Hensman, R. Mills, S.G. Pierce, K. Worden, M. Eaton, Locating acoustic emission sources in complex structures using Gaussian processes. Mechanical Systems and Signal Processing 24(1): 211-23(2010)

[7] W.H. Prosser, M.A. Hamstad, J. Gary, A. O'Gallagher, Reflections of AE waves in finite plates: finite element modeling and experimental measurements. Journal of Acoustic Emission 17: 37-47 (1999)

[8] A. Labib, D. Kennedy, C.A. Featherston, Crack localization in frames using natural frequency degrations, Computers and structures, vol.157, pp.51-59 (2015)

[9] D. Aljets, A. Chong, S. Wilcox, K. Holford, Acoustic emission source location in plate like structures using a closely arranged triangular sensor array. Journal of Acoustic Emission 28: 85-98 (2010)

[10] X. Zhang, E. Song, H. Liu, B. Li , Acoustic Source Localization via Subspace Based Method Using Small Aperture MEMS Arrays, Journal of Sensors, Vol. 2014 (2014), Article ID 675726, 14 pages, http://dx.doi.org/10.1155/2014/675726

[11] F. Bahram, Fracture mechanics of metals, composites, welds and bolted joints, Springer science and business media, DOI 10.1007/978-1-4615-1585-2, (2001)

[12] D.A. Axinte, D.R. Natarajan, , N.N. Gindy, An approach to use an array of three acoustic emission sensors to locate uneven events in machining-Part 1: method and validation. International Journal of Machine Tools and Manufacture 45(14): 160513(2005)

[13] S. Sengupta, A. Kumar Datta, P. Topdar, Structural damage localisation by acoustic emission technique: A state of the art review, Latin American journal of solid structures, vol.12, pp.1562-1585 (2015)

[14] M.G.R. Sause, S. Horn, Simulation of lamb wave excitation for different elastic properties and acoustic emission source geometries. Journal of Acoustic Emission 28: 109-21(2010)

[15] H. Suzuki, T. Kinjo, Y. Hayashi , M. Takemoto, K. Ono, Y. Hayashi,. Wavelet transform of acoustic emission signals. Journal of Acoustic Emission 14(2): 69-84 (1996)

[16] M. Takemoto, H. Nishino, K. Ono, Wavelet transform-applications to AE signal analysis. Acoustic Emis-sion-Beyond the Millennium: 35-56 (2000)

[17] M.A. Hamstad, A. O'Gallagher, J. Gary, A wavelet transform applied to acoustic emission signals: Part 2: Source Location. Journal of Acoustic Emission 20: 62-82(2002b)

[18] A. Mostafapour, S. Davoodi, M. Ghareaghaji, Acoustic emission source location in plates using wavelet analysis and cross time frequency spectrum. Ultrasonics 54(8): 2055$62(2014)$

[19] Miller, R.K., Hill, E.v.K., and Moore, P.O., Nondestructive Testing Handbook, 3rd Ed., Vol. 6. Acoustic Emission Testing. Columbus, OH: American Society for Nondestructive Testing, 2005, p. 32 
[20] N. Herausgeber, R. Ganesh, W. Wang, Blind Source Separation, Springer Series in Signals and Communication Technology (2014)

[21] Z. Nazarchuk, V. Skalskyi, O. Serhiyenko, Acoustic Emission: Methodology and Application, Springer Serie: Foundations of Engineering Mechanics, 2017, Print ISBN: 978-3-319-49348-0, Electronic ISBN: 978-3-319-49350-3

[22] R. Ibrahim, Handbook of structural life assessment, Jojn Wiley\&Sons, 2017

[23] B. Lawn, Fracture of Brittle Solids, 2nd Ed., Cambridge University Press, (1997).

[24] K. Wasmer, C. Ballif, C. Pouvreau, D. Schulz, and J. Michler, Dicing of GalliumArsenide High Performance Laser Diodes for Industrial Applications: Part II: Cleavage Operation, Journal of Materials Processing Technology, Vol. 198, Issues 1-3, pp: 105-113 (2008).

[25] K.Wasmer, C. Ballif, C. Pouvreau, D. Schulz, and J. Michler, "Dicing of GalliumArsenide High Performance Laser Diodes for Industrial Applications: Part I: Scratching Operation", Journal of Materials Processing Technology, Vol. 198, Issues 1-3, pp: 114$121(2008)$

[26] Y.N. Kulchin, O.B. Vitrik, A.V. Dyshlyuk, I.N. Nemov, Differential reflectometry of FBG sensors in the wide spectral range, Laser physics 21 (2), pp.304-307 (2011)

[27] I. Daubechies I., Ten Lectures on Wavelets; CBMS-NSF Lecture Notes nr. 61, SIAM (1992)

[28] A. Gupta, S.D. Joshi, S. Prasdad, A new approach for statistically matched wavelet, IEEE Signal processing, vol.53,no.5, pp.1778-1781(2005)

[29] T. Lin, S. Xu, Q. Shi, and P. Hao. An algebraic construction of orthonormal M-band wavelets with perfect reconstruction, Applied mathematics and computation, 172(2):717730 (2006)

[30] H.F. Trotter, An elementary proof of the central limit theorem. Arch Math; 10:226234 (1959)

[31] A. Hyvärinen, J. Karhuen, E. Ora, Independent component analysis, John Whiley\&Sons, New York, (2001)

[32] A. Singer, R. Coifman, Non-linear independent component analysis with diffusion maps, Appl. Comput. Harmon. Anal., vol.25,pp.226-239, (2008)

[33] J. Velasco, D. Pizarro, J. Macias-Guarasa, Source Localization with Acoustic Sensor Arrays Using Generative Model Based Fitting with Sparse Constraints. Sensors (Basel, Switzerland). 12(10):13781-13812 (2012)

[34] D. Marquardt: An Algorithm for Least-Squares Estimation of Nonlinear Parameters, SIAM J. Appl. Math. 11, 431-441, (1963) 